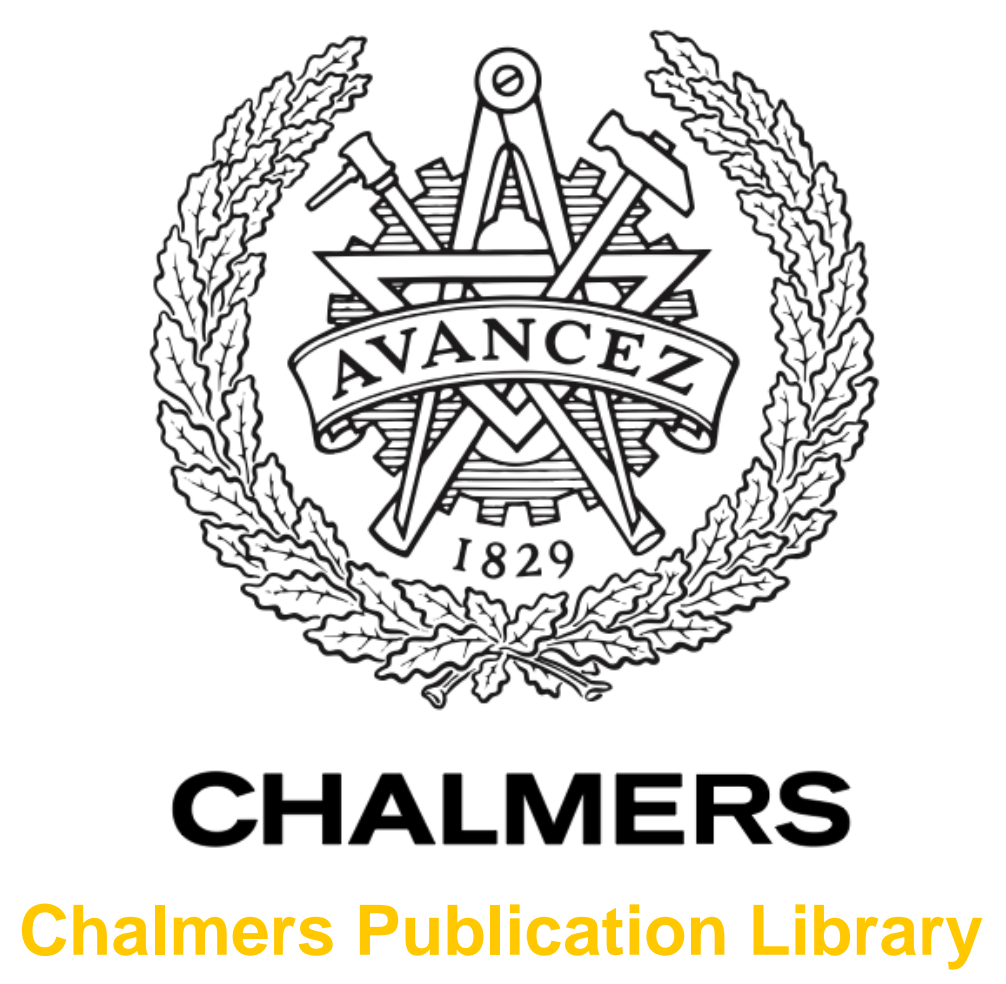

Concurrent Design and Control of Automated Material Handling Systems

This document has been downloaded from Chalmers Publication Library $(\mathrm{CPL})$. It is the author's version of a work that was accepted for publication in:

Proc. 9th IEEE International Conference on Automation Science and Engineering (CASE 2013), Madison Wisconsin, August

Citation for the published paper:

Mashaei, M. ; Lennartson, B. (2013) "Concurrent Design and Control of Automated Material Handling Systems". Proc. 9th IEEE International Conference on Automation Science and Engineering (CASE 2013), Madison Wisconsin, August

Downloaded from: http://publications.lib.chalmers.se/publication/181004

Notice: Changes introduced as a result of publishing processes such as copy-editing and formatting may not be reflected in this document. For a definitive version of this work, please refer to the published source. Please note that access to the published version might require a subscription. 


\title{
Concurrent Design and Control of Automated Material Handling Systems
}

\author{
Maziar Mashaei ${ }^{1,2}$, Bengt Lennartson ${ }^{1}$ \\ ${ }^{1}$ Department of Signals and Systems, Chalmers University of Technology, Sweden \\ ${ }^{2}$ FlexLink AB, Göteborg Sweden
}

\begin{abstract}
Lean and agile design and control of an automated material handling system are investigated in this paper. The demands for a minimal number of handling resources and their maximal utilization emphasize the importance of a concurrent structure and control design for a handling mechanism in the conceptual phase. To provide this concurrency, a universal model based on mathematical linear constraints is developed to define a set of part movements without concerning a specific handling technology. Furthermore, an objective characterizing optimal part movements, according to the lean and agile paradigms, is formulated in the conceptual design phase. Control measures, which are obtained by solving the mixed integer linear model including the objective and constraints, provide important keys for designers to conceptualize a proper design of an automated material handling system. To show the application of developed approach, a case study is presented and discussed.
\end{abstract}

Key words: Automated material handling system, Concurrent design and control, Lean and agile paradigms, Mixed integer linear optimization model.

\section{INTRODUCTION}

Handling, buffering, and locating actions are the most frequent non-value adding activities in a manufacturing plant. The resources, which perform these functions and receive commands from electronic control devices, are categorized in a group of Automated Material Handling Systems (AMHSs). The major examples of AMHSs are automated guided vehicles (AGVs), pallet and conveyors systems, cranes, and handling robots.

Operational and economical issues are the main concerns in the design and control of a manufacturing plant. Defining waste as a resource or effort that does not transform a part (does not add any value to a part), Lean philosophy [1] sums up these issues with the concept of waste minimization. According to this philosophy, a material handling system is an auxiliary system, because it does not transform a part, but it supports adding value activities. Besides, according to Agile philosophy [1] an AMHS should be flexible enough to cope with various production scenarios. Hence, the lean and agile design of an AMHS that captures the minimum number of resources and the minimal cost, while fulfilling different production scenarios with a maximum flow efficiency is a vision for designers.

The realization of this vision is a great challenge because there is a trade-off between maximum resource utilization and maximum flow efficiency. The less number of handling resources is devised, the less probable it is to obtain a high flow efficiency. In the literature this challenge has been broken into two subproblems; control design and system (plant) design.

The main issue in the control design is to maximize flow efficiency with a nearly fixed number of handling resources and technologies (the number of carriers such as pallets and AGVs may be variable). Flow enhancement, [5], [6], [7], [8], makespan minimization [9], [10], reduction of the cycle time and Work-In-Process (WIP), [11], [12], [13], [14] are some instances for the control design. On the other hand, the main objective in the plant design is to minimally design a handling system (maximize handling resource utilization) for a given flow rate, [2], [3], [4].

A sequential procedure of system and control design is a classical approach in the development of a material handling system. Although this approach has been applied in practice and enhanced in a course of years, there are still some evidences showing a vast gap between a practical design and the visionary one based on the lean and agile philosophies. According to [2], [15], in practice large percentages of operational cost (20\% to $50 \%)$, factory space $(55 \%)$, and production time $(87 \%)$ of a manufacturing sector are attributed to its material handling system. These values show that large portions of capital, time, and effort are associated with nonvalue adding activities that can highly affect product costs (15\% to $70 \%)$, [2].

One possible reason for this gap can be the lack of concurrent structure and control design for AMHSs. In the classical approach, the structure and resources of an AMHS are first specified, sacrificing the precise modeling of the system dynamics. Then for the given structure, control set points are calculated as addressed in the aforementioned studies. This approach does not guarantee a lean and agile design for AMHSs, because the impact of control parameters on the structure design is not fully and precisely investigated.

In this paper we address a novel approach for the realization of an AMHS based on the integration of the system and control problems in one design framework. The concurrent system and control design framework promisees finding solutions for both issues; the maximum utilization and the maximum flow efficiency (lean and agile design of an AMHS), which can not be obtained by using the classical sequential design approach. 


\section{LEAN STRUCTURE AND CONTROL DESIGN OF AN} AMHS

An AMHS can be modeled by a graph in which locating, connecting, and intersection positions are represented by nodes, and flow paths are specified by edges. For example, Fig. 1 shows a complete graph of a system with 6 nodes to conduct and locate parts for operating machines $M 1$ to $M 4$.

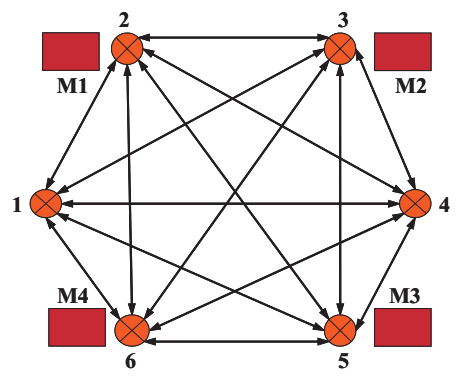

Fig. 1. A complete graph model of an AMHS, including 6 nodes.

Regarding the graph model, the structural design procedure should specify the number and position of nodes, technology and equipment type for each node, number and configuration of edges, and flow direction and handling mechanism for each edge. On the other hand, the control design procedure may characterize the number of parts, the velocity of parts in each flow path, the buffer level and queuing policy in each edge, scheduling and time instances for releasing parts in each node.

Based on the graph model and without concerning a handling technology, an index for measuring the lean design can be defined according to the numbers of nodes, edges, and carriers. The lower these numbers are, the fewer resources are integrated in the system. This implies a procedure for the conceptual design phase that takes a complete graph as its input and provides a graph with a lower number of nodes, edges, and WIP as its output. Naming this procedure as the lean filter, the maximum reduction is achieved when the output of the filter is a directed simple loop with the minimal number of nodes and WIP. In this case the nodes only represent locating positions for machines. For instance, Fig. 2 demonstrates a reduction of the complete graph in Fig. 1 to the directed loop with 4 nodes.

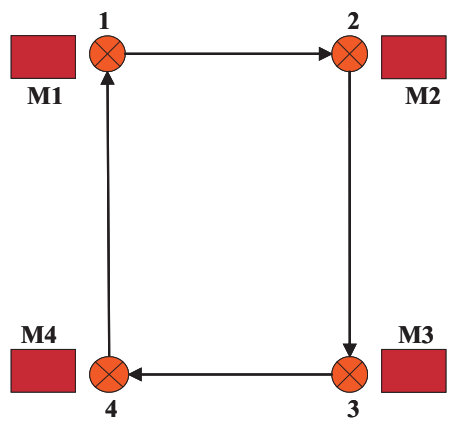

Fig. 2. Directed loop model of the AMHS with 4 nodes.

Although the one-loop design may have the lowest number of resources, it cannot be applied if the desired production rate is not fulfilled. The major problem in this design is that the flow times of parts in the loop can be significantly increased due to the rise of unnecessary transportation and waiting times. As a result, the average throughput $T H$ decreases according to the Hopp and Spearman equation

$$
T H=\frac{W I P}{F T}
$$

which relates $T H$ to the average flow time $F T$ and work-inprocess $W I P$, [16]. One solution to this problem is to increase $W I P$ in order to maintain the desired $T H$. But this solution can not always be practised because a part carrier could be the most expensive resource in an AMHS (for example AGV), or there could be a barrier for increasing WIP (limited buffer spaces). Having these limitations, a more complicated configuration should be designed for the AMHS to satisfy the desired throughput. To make this more clear, the following example is devised.

The configuration shown in Fig. 2 is used to transport two part types $\mathrm{A}$ and $\mathrm{B}$ between 4 processing machines. The part operations in the machines are:

- $A: O_{1}\left(M^{1}\right) \rightarrow O_{2}\left(M^{2}\right) \rightarrow O_{3}\left(M^{3}\right) \rightarrow O_{4}\left(M^{2}\right) \rightarrow O_{5}\left(M^{4}\right)$

- $B: O_{1}\left(M^{1}\right) \rightarrow O_{2}\left(M^{2}\right) \rightarrow O_{3}\left(M^{3}\right) \rightarrow O_{4}\left(M^{4}\right)$

This means that part A circulates the loop twice and part $\mathrm{B}$ once. Hence, the flow paths of A and B can be expressed based on the nodes as

- $A: 1 \rightarrow 2 \rightarrow 3 \rightarrow 4 \rightarrow 1 \rightarrow 2 \rightarrow 3 \rightarrow 4$

- $B: 1 \rightarrow 2 \rightarrow 3 \rightarrow 4$

If for any reason the desired throughput cannot be achieved by using the one-loop design, the system configuration should be modified to reduce the flow paths and related flow times. One solution is to introduce a feedback from $M 3$ to $M 2$ for part A. This solution is depicted in Fig. 3 by introducing two extra nodes and three extra edges.

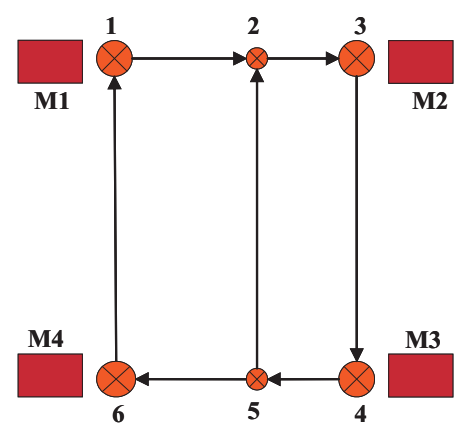

Fig. 3. Modified graph of the AMHS with 6 nodes. The small nodes represent the intersections between the paths.

The big nodes indicate the locating points, at which the parts are positioned for operating actions, and the small nodes represent the intersections between the paths. Based on this modification the flow paths are changed to

- $A: 1 \rightarrow 2 \rightarrow 3 \rightarrow 4 \rightarrow 5 \rightarrow 2 \rightarrow 3 \rightarrow 4 \rightarrow 5 \rightarrow 6$

- $B: 1 \rightarrow 2 \rightarrow 3 \rightarrow 4 \rightarrow 5 \rightarrow 6$

In practice, the added nodes and edges imply resources such as fixture, robot, conveyor, aisle, diverter, merger etc., where the types completely depend on the handling technology. 
Which type and what number of resources should be added to a one-loop AMHS to maintain a desired throughput are challenging questions regarding the lean design. In the next section, we introduce a measure and a design framework to give some clues to tackle these challenges.

\section{UNIVERSAL MODEL OF AN AMHS}

A practical handling mechanism has limitations on transportation time, queuing policy, and buffering and locating parts. For example, a handling equipment may not be faster than $1 \mathrm{~m} / \mathrm{s}$, it follows First Input First Output (FIFO) queuing policy, and it may only buffer two parts for locating actions. If these limitations are dropped, large domains can be specified for variations of WIP and FT. Hence, with such relaxations on part velocities, buffer capacities, and queuing policies, theoretically it is not necessary to add more nodes and edges to the one-loop AMHS model to fulfill a desired throughput. A one-loop AMHS configuration with these theoretical relaxations realizes a universal model for any handling system technology.

The dynamics analysis of parts in the universal model provides metrics for the relaxed parameters. If these metrics are in practical ranges, then the one-loop AMHS design is also practically possible. Otherwise, extra physical resources should be added to the one-loop design in order to translate the unrealistic metrics from theory into practice. For example, assume that the transportation time of a part between two the nodes 2 and 3 in Fig. 2 is calculated to be zero seconds. In theory, this means that the length between the two nodes is zero, or/and the part velocity goes to infinity. In practice; however, both of these assumptions are invalid. Thus, to obtain an applicable shorter transportation time, some modifications should be applied to the system configuration. Providing that there is no process for a part in M3, an extra edge (bypass edge) between the nodes 2 and 4 may present such a realization.

A proper translation of the metrics into physical resources requires a large amount of knowledge, experience, and engineering skills about the system technology and architecture. In other words, there is no formal method (language) for this translation. However, it is possible to formally define criteria for the metrics, leading to the minimal number of resources for the translation. In this section, these criteria are introduced, and a mathematical model which defines the minimal number of handling resources is developed.

\section{A. Notations and assumptions for the universal model}

In the universal model, the number of edges is the same as the number of nodes. Hence, the model can be partitioned into segments so that each includes one edge and its following node. Based on this partitioning, the following notations are defined:

- $\ell \in M=\{1,2, \ldots, m\}$ is the index over all segments.

- $N^{\ell}(t)$ is the number of carriers at time $t$ in segment $\ell$.

- $b^{\ell}$ is the buffer size of segment $\ell$.

- $L^{\ell}$ is the length of segment $\ell$.
- $i \in I=\{1, \ldots, n\}$ is the index over part instances in the ordered part set $\left\langle P_{1}, P_{2}, \ldots P_{n}\right\rangle$.

- $v_{i}^{\ell}[\mathrm{m} / \mathrm{s}]$ denotes the velocity of carrier for $P_{i}$ in segment $\ell$. Furthermore, $T_{c_{i}}^{\ell}=1 / v_{c_{i}}^{\ell}$ is the transportation time of $P_{i}$, passing 1 meter in segment $\ell$.

- $T_{r_{i}}^{\ell}=L^{\ell} T_{c_{i}}^{\ell}$ is the transportation time of $P_{i}$ in segment $\ell$.

- $\tau_{i}^{\ell}$ is the locating time of $P_{i}$ for some operation in segment $\ell$ (the setup time is also included in the locating time).

- $W_{i}^{\ell}$ is the waiting time of $P_{i}$ in the buffer of segment $\ell$.

- $F T_{i}^{\ell}=T_{r_{i}}^{\ell}+\tau_{i}^{\ell}+W_{i}^{\ell}$ is the flow time of $P_{i}$ in segment $\ell$.

In addition to the notations, some assumptions are needed to approximately represent complex functions in an AMHS on mathematical form. These assumptions are introduced for the model.

Deterministic system: Processing times $\tau_{i}^{\ell}$ and transportation times $T_{r_{i}}^{\ell}$ are deterministic and constant.

Cyclic system: A part set containing $n$ parts $\left\{P_{1}, P_{2}, \ldots P_{n}\right\}$ is cyclically fed to the segments from the loading segment $\ell=1$ to the unloading segment $\ell=m$. The cycle number, $k$, specifies the part set fed in $k^{\text {th }}$ cycle. Each segment handles $k^{\text {th }}$ part set before $(k+1)^{t h}$ part set. Additionally, the order $i \in I$, by which $P_{j}$ in the set is released from the segment $\ell$, is constant over time and indicated by the releasing vector $I^{\ell}$. This means that $i^{t h}$ component of this vector is $j$ or $I_{i}^{\ell}=j$. Accordingly, the notation $I^{\ell}(j)=i$ is used to specify the order of part $j$ released in the segment $\ell$.

CONWIP system: Fixed number of carriers or CONstant Work in Process is considered in the system $\left(N=\sum_{\ell=1}^{m} N^{\ell}(t)\right)$.

Availability of parts and tools: Parts are always available to be loaded in the first segment, and when a part is located for an operation, the related tools are available.

Stationary behavior: If a part should circulate the loop more than once (re-entrance behavior), then for each circulation a distinct type instance is given to the part. In the system stationary state, furthermore, it is assumed that all type instances related to a part are fed to the system as different parts in the part set.

\section{B. Mathematical model of the lean design criterion}

Based on the introduced notations and assumptions, we have

$$
W I P=N, \quad T H=\frac{n}{C}, \quad F T=\frac{\sum_{\ell=1}^{m} \sum_{i=1}^{n} F T_{i}^{\ell}}{n}
$$

where $N$ is the number of carriers in the system and $C$ is the cycle time for the production of the part set $\left\{P_{1}, P_{2}, \ldots P_{n}\right\}$. Accordingly, (1) can be reformulated as

$$
N C=\sum_{\ell=1}^{m} \sum_{i=1}^{n} T_{r_{i}}^{\ell}+\tau_{i}^{\ell}+W_{i}^{\ell}
$$


In this equation, the cycle time $C$ and operation times $\tau_{i}^{\ell}$ are given and the other terms are variables.

The lean design of an AMHS should realize the minimal number of resources satisfying (2). Assuming that $\Re$ is the index measuring the number of resources in the AMHS, the lean design can mathematically be expressed by the optimization model:

$$
\begin{aligned}
& \min \Re \\
& \text { subject to } \\
& N C=\sum_{\ell=1}^{m} \sum_{i=1}^{n} T_{r_{i}}^{\ell}+\tau_{i}^{\ell}+W_{i}^{\ell}
\end{aligned}
$$

The objective function $\Re$ is developed according to the design variables as follows:

Number of carriers: The equation

$$
\Re_{1}(N)=N
$$

is a simple measure for the number of carriers.

Buffer size: The reduction of a segment buffer size may save space and some resources in an AMHS design. Hence, the index

$$
\Re_{2}=\sum_{\ell=1}^{m} b^{\ell}
$$

is defined to measure these related resources.

Carrier (part) velocities: Large values of part velocities $\left(v_{i}^{\ell}\right)$, outside practical ranges, and high variations of these values may increase the number of resources such as electric motors, conveyors, aisles, spaces, and robots. They also increase the complexity of the motion control for various part instances. Therefore, to give a measure based on these parameters, it is reasonable to define

$$
\Re_{3}=V^{U}+\sum_{\ell=1}^{m} \sum_{i=1}^{n}\left(v_{i}^{\ell}-V^{L}\right)
$$

where $V^{L}$ and $V^{U}$ are the lower and upper boundaries for the velocities $\left(V^{L} \leq v_{i}^{\ell} \leq V^{U}\right)$. The term $T_{r_{i}}^{\ell}$ in the constraint (2) is equal to $L^{\ell} T_{c_{i}}^{\ell}$ for $T_{c_{i}}^{\ell}=1 / v_{i}^{\ell}$. Hence, (6) is not linear based on (2). A linear form of the measure is achieved by the new definition

$$
\Re_{3}=\sum_{\ell=1}^{m} \sum_{i=1}^{n}\left(T_{c}^{U}-T_{c_{i}}^{\ell}\right)-T_{c}^{L}
$$

where, $T_{c}^{L}=1 / V^{U}$ and $T_{c}^{U}=1 / V^{L}\left(T_{c}^{L} \leq T_{c_{i}}^{\ell} \leq T_{c}^{U}\right)$. It should be noted that (7) can not be formulated as a result of (6). Obviously, this term gives a linear form of measuring resources based on part transportation times (rather than part velocities).

Part scheduling: There are two approaches to affect the waiting time of $P_{i}$ in segment $\ell$. Change the schedule for the releasing action of $P_{i}$ in the segment (change $I_{i}^{\ell}$, see Cyclic system in Section III-A) and/or make a tunneling action for $P_{i}$ if there is no process for this part in segment $\ell$. The tunneling action means that when another part is being located for an operation in segment $\ell, P_{i}$ bypasses the locating position (and the other part) and enters segment $\ell+1$. Integrating scheduling and tunneling mechanisms in an AMHS is often expensive and demands smart controls and related resources. For example, take a conveyor line where parts enter and exit with FIFO queuing policy. This handling mechanism is simple because it is not necessary to change the queuing positions of parts in the line. If there is such a necessity, extra resources such as diverter, merger, or robot may be devised in the line. Hence, it is crucial to minimize the number of scheduling changes and tunneling actions to reduce the number of resources in an AMHS. This is measured by the term

$$
\mathfrak{R}_{4}=\sum_{\ell=2}^{m} \sum_{i=1}^{n}\left|I_{i}^{\ell}-I_{i}^{\ell-1}\right|+\sum_{\ell=1}^{m} \sum_{i=1}^{n} \beta_{i}^{\ell}
$$

for

$$
\beta_{i}^{\ell}=\left\{\begin{array}{l}
1: \text { if there is a tunneling action for } P_{i} \text { in segment } \ell \\
0: \text { otherwise }
\end{array}\right.
$$

Introducing constraints $\alpha_{i}^{\ell} \geq I_{i}^{\ell}-I_{i}^{\ell-1}$ and $\alpha_{i}^{\ell} \geq I_{i}^{\ell-1}-I_{i}^{\ell}$, a linearized form of $\Re_{4}$ can be obtained as

$$
\Re_{4}=\sum_{\ell=2}^{m} \sum_{i=1}^{n} \alpha_{i}^{\ell}+\sum_{\ell=1}^{m} \sum_{i=1}^{n} \beta_{i}^{\ell}
$$

Realization of $\mathfrak{R}$ : The objective function in (3) is determined by

$$
\Re=\sum_{e=1}^{4} w_{e} \Re_{e}
$$

where $\mathfrak{R}_{e}$ for $e \in\{1,2,3,4\}$ respectively denote the relations (4), (5), (7), and (10), and $w_{e}$ are weight coefficients. Introducing

$$
\begin{aligned}
& \Re_{e}^{\min }=\min \Re_{e} \\
& \text { subject to } \\
& N C=\sum_{\ell=1}^{m} \sum_{i=1}^{n} T_{r_{i}}^{\ell}+\tau_{i}^{\ell}+W_{i}^{\ell}
\end{aligned}
$$

the coefficients are calculated by $w_{e}=\rho_{e} / \hat{\mathfrak{R}}_{e}^{\min }$, where $\rho_{e} \in$ $[0,1]$ is a coefficient showing the tendency to a type of handling technology, and $\hat{\mathfrak{R}}_{e}^{\text {min }}$ is defined by

$$
\hat{\mathfrak{R}}_{e}^{\text {min }}= \begin{cases}\left|\mathfrak{R}_{e}^{\text {min }}\right| & : \text { if }\left|\mathfrak{R}_{e}^{\text {min }}\right| \geq 1 \\ 1 & : \text { if }\left|\mathfrak{R}_{e}^{\text {min }}\right|<1\end{cases}
$$

Here the condition $\left|\Re_{e}^{\min }\right|<1$ is considered to bound $w_{e}$ in $[0,1]$. For example, if there is a high tendency to use AGVs, then $\rho_{1}=1$ and other $\rho_{e}<1 ; e \neq 1$, because the number of carriers plays an important role in the cost of AGV systems. If there is no prioritized AMHS technology, $\rho_{e}=1$ for all weight coefficients.

To properly develop the optimization models (3) and (12), the terms $T_{r_{i}}^{\ell}$ and $W_{i}^{\ell}$ in (2) should also be related to the design variables. Unlike $T_{r_{i}}^{\ell}=L^{\ell} T_{c_{i}}^{\ell}$, the term $W_{i}^{\ell}$ can not be explicitly presented as a function of the design variables. To relate $W_{i}^{\ell}$ to the decision variables, the mathematical constraints characterizing (2) are developed in the next section. 


\section{Design COnStRaint MOdels}

Handling time of a part instance in the universal model is subject to various constraints, due to scheduling, cyclic, dynamic, CONWIP, and buffer criteria. Equation (2) is too abstract to express all these criteria. Hence, the mapping of this equation to a set of mathematical models characterizing the denoted criteria seems necessary. In this section, we do so and develop the mathematical models denoted as design constraints. In the first step of developing these constraints, a relation between the number of carriers in the model and the individual flow time of a part instance is developed.

\section{Notations:}

- $k \in K=\left\{1,2, \ldots k^{s}, k^{s}+1, \ldots, k_{U}^{s}\right\}$ is the index over cycles in transient and steady state phases. Moreover, $K^{s}=\left\{k^{s}, k^{s}+1, \ldots, k_{U}^{s}\right\}$ includes all index cycles, in which the system is in the steady state phase.

- $\hat{S}_{j}^{\ell}[k]$ is the time when $P_{j}$ in $\left\{P_{1}, P_{2}, \ldots P_{n}\right\}$ is released by segment $\ell$ in cycle $k$.

- $S_{i}^{\ell}[k]$ is the time when a part in the ordered part set $\left\langle P_{1}, P_{2}, \ldots P_{n}\right\rangle$ is released by segment $\ell$ with order $i$ in cycle $k$.

- $C_{i}^{\ell}[k]=S_{i+1}^{\ell}[k]-S_{i}^{\ell}[k] \quad$ for $\quad i \in I \backslash\{n\} \quad$ and $C_{n}^{\ell}[k]=S_{1}^{\ell}[k+1]-S_{n}^{\ell}[k]$ are defined as interval times.

Proposition 1: In the universal model, the relation between the number of carriers $N$, the flow time (lead time) of $P_{j}, F T_{j}=\sum_{\ell=1}^{m} F T_{j}^{\ell}$, and the interval times $C_{g(i, e)}^{m}[k]$ is set by equations:

$$
\begin{gathered}
F T_{j}[k]=\sum_{e=1}^{N_{i}} C_{g(i, e)}^{m}\left[k+f\left(i, e, N_{i}\right)\right] \\
n N=\sum_{i=1}^{n} N_{i} \\
f(i, e, x)=\left\lfloor\frac{((i-x) \bmod n)-e}{n}\right\rfloor
\end{gathered}
$$

and

$$
g(i, e)=\left\{\begin{array}{lrl}
n: & i-e=n k, k \in \mathbb{Z} \\
(i-e) \bmod \quad n: & i-e \neq n k, k \in \mathbb{Z}
\end{array}\right.
$$

where $N_{i}$ is the number of parts released by the segment $m$, including the last part, $P_{j}$, with the order $i=I^{m}(j)$.

Proof: The order of releases for $P_{j}$ in the segments can be represented by the vector $\left[I^{1}(j), I^{2}(j), \ldots, I^{m}(j)\right]^{T}$. Assume $P_{j}$ is the part that is now entering the system with the carrier which was handling the part $P_{j}$ with the order of $\hat{i}=I^{m}(\hat{j})$. Now consider that $N_{i}$ parts (including $P_{j}$ ) should exit from the line until $P_{j}$ is released by the segment $m$ with the order $i=I^{m}(j)$. Hence,

$$
i=\left(\hat{i}+N_{i}\right) \bmod n
$$

and

$$
N_{i}=N+\sum_{\ell=2}^{m}\left(I^{\ell}(j)-I^{\ell-1}(j)\right)=N+I^{m}(j)-I^{1}(j) \geq 1
$$

which implies that the change of order cannot exceed $N$. Moreover, we have

$$
F T_{j}[k]=\sum_{e=1}^{N_{i}} C_{y(\hat{i}, e)}^{m}\left[k+h\left(\hat{i}, e, N_{i}\right)\right]: \hat{i}, j \in I
$$

where

$$
y(\hat{i}, e)= \begin{cases}n: & \hat{i}+e-1=n k, k \in \mathbb{Z} \\ (\hat{i}+e-1) \bmod n: & \text { otherwise }\end{cases}
$$

and

$$
h\left(\hat{i}, e, N_{i}\right)=\left\lfloor\frac{\hat{i}+e-1-N_{i}}{n}\right\rfloor
$$

Equation (19) leads to

$$
\sum_{i=1}^{n} N_{i}=n N+\sum_{j=1}^{n}\left(I^{m}(j)-I^{1}(j)\right)=n N
$$

On the other hand, defining $\hat{e}=N_{i}-e+1$, and using (18), we have $g(i, \hat{e})=y(\hat{i}, e)$ and $f\left(i, \hat{e}, N_{i}\right)=h\left(\hat{i}, e, N_{i}\right)$. This implies

$$
\sum_{e=1}^{N_{i}} C_{y(\hat{i}, e)}^{m}\left[k+h\left(\hat{i}, e, N_{i}\right)\right]=\sum_{\hat{e}=N_{i}}^{1} C_{g(i, \hat{e})}^{m}\left[k+f\left(i, \hat{e}, N_{i}\right)\right]
$$

which gives (14) according to (20).

Compared to (2), the relation (14) gives more information about the dynamic behavior of an individual part instance. But this information is not yet enough to realize the waiting times $W_{i}^{\ell}$. To obtain the desired information, relations between the part flow times $F T_{i}^{\ell}[k]$ and part releasing times $S_{i}^{\ell}[k]$ should be mathematically defined. This leads to the following design constraints.

Scheduling constraints: The segment releasing vector $I^{\ell}$ is generated from $J=[1,2, \ldots n]$ by $I^{\ell}=\Delta^{\ell} J^{T}$, where $\Delta^{\ell}$ is an $n \times n$ permutation matrix. Denoting $\left[\Delta^{\ell}\right]_{i, j}=\delta_{i, j}^{\ell}$ and $I_{i}^{\ell}=\sum_{j=1}^{n} \delta_{i, j}^{\ell} j$, each operation time $\tau_{j}^{\ell}$ based on the order $j$ can be mapped to the operation time $T_{i}^{\ell}$ based on the order $I^{\ell}$ as follows

$$
\begin{aligned}
& i, j \in I, \quad \ell \in M \\
& \sum_{i=1}^{n} \delta_{i, j}^{\ell}=1, \quad \sum_{j=1}^{n} \delta_{i, j}^{\ell}=1, \quad \sum_{j=1}^{n} \delta_{i, j}^{\ell} \tau_{j}^{\ell} \leq T_{i}^{\ell}
\end{aligned}
$$

Cyclic and periodic behavior constraints: These constraints demand two crucial properties for the universal model. The first property is the cyclic manner of part releasing actions in a segment with the order $I^{\ell}$ or $S_{i+1}^{\ell}[k]-S_{i}^{\ell}[k]=$ $C_{i}^{\ell}[k]$. The second property forces the periodic behavior $C_{i}^{\ell}[k]=C_{i}^{\ell}$ in the stationary state. To tune the cycle time $C$, then $\sum_{i=1}^{n} C_{i}^{m}[k]=C$, and for $\ell \neq m$ the condition can be relaxed to $\sum_{j=1}^{n} \tau_{j}^{\ell} \leq \sum_{i=1}^{n} C_{i}^{\ell}[k] \leq C$. Therefore,

$$
\begin{aligned}
& k \in K^{s}, \ell \in M \\
& S_{i+1}^{\ell}[k]-S_{i}^{\ell}[k]=C_{i}^{\ell} \quad: \quad i \in I \backslash\{n\} \\
& S_{1}^{\ell}[k+1]-S_{n}^{\ell}[k]=C_{n}^{\ell}: \quad k \in K^{s} \backslash\left\{k_{U}^{s}\right\} \\
& \sum_{i=1}^{n} C_{i}^{m}=C \\
& \sum_{j=1}^{n} \tau_{j}^{\ell} \leq \sum_{i=1}^{n} C_{i}^{\ell \leq C}: \quad \ell \in M \backslash\{m\}
\end{aligned}
$$


Segment dynamic behavior constraints: The duration of $P_{j}$ in segment $\ell, \hat{S}_{j}^{\ell}[k]-\hat{S}_{j}^{\ell-1}[k]$, is bounded by the constraint $\hat{S}_{j}^{\ell}[k]-\hat{S}_{j}^{\ell-1}[k] \geq \tau_{j}^{\ell}+T_{r_{j}}^{\ell}$. The inequity indicates the fact that there might be a waiting time for this part in the denoted duration. If the part set $\left\{P_{1}, P_{2}, \ldots P_{n}\right\}$ enters to the segment $\ell$ by the order $I^{\ell-1}$ and releases from this segment by the order $I^{\ell}$, the constraint based on the order of part $j$ in $I^{\ell-1}$ and $I^{\ell}$ is modified as

$$
\begin{aligned}
S_{I^{\ell}(j)}^{\ell}[k]-S_{I^{\ell-1}(j)}^{\ell-1}[k] & \geq\left(\delta_{I^{\ell}(j), j}^{\ell}+\delta_{I^{\ell-1}(j), j}^{\ell-1}-2\right) \Phi \\
& +T_{I^{\ell}(j)}^{\ell}+L^{\ell} T_{C_{I^{\ell}(j)}^{\ell}}
\end{aligned}
$$

or

$$
S_{i}^{\ell}[k]-S_{\hat{i}}^{\ell-1}[k] \geq\left(\delta_{i, j}^{\ell}+\delta_{\hat{i}, j}^{\ell-1}-2\right) \Phi+T_{i}^{\ell}+L^{\ell} T_{c_{i}}^{\ell}
$$

where $\Phi$ is a large constant number (larger than $n F T$ ), $I^{\ell}(j)=i$, and $I^{\ell-1}(j)=\hat{i}$. On the other hand, the relation $S_{i}^{\ell}[k]-S_{i-1}^{\ell}[k] \geq T_{i}^{\ell}$, without concerning $P_{i}$ tunneling behavior, is achieved. In order to include the part tunneling behavior in this relation, first we introduce the permissibility tunneling factor of $P_{j}$ in segment $\ell$ as

$$
\varepsilon_{j}^{\ell}= \begin{cases}1: & \tau_{j}^{\ell}>0 \\ 0: & \tau_{j}^{\ell}=0\end{cases}
$$

$\varepsilon_{j}^{\ell}=1$ means that there is no tunneling action for $P_{j}$ in the segment. In other words, a part does not bypass the locating position if there is a non-zero operating time of the part in the segment. Now, the relation $S_{i}^{\ell}[k]-S_{i-1}^{\ell}[k] \geq T_{i}^{\ell}$ is modified to the constraints

$$
\begin{aligned}
& S_{i}^{\ell}[k]-S_{i-e}^{\ell}[k]+\beta_{i-e}^{\ell} \Phi \geq T_{i}^{\ell}: e=\{1, \ldots, i-1\} \\
& 1-\sum_{j=1}^{n} \delta_{i, j}^{\ell} \varepsilon_{j}^{\ell} \geq \beta_{i}^{\ell}
\end{aligned}
$$

where $\beta_{i}^{\ell} \geq 0$ is a binary variable. This variable is set to zero if $\varepsilon_{j}^{\ell}=1$ or $\alpha_{i}^{\ell}=0$. Otherwise, $\beta_{i}^{\ell}$ may be set to one to relax the constraint $S_{i+1}^{\ell}[k]-S_{i}^{\ell}[k] \geq T_{i+1}^{\ell}$. Considering (26), (29), and the assumption that the handling of a part set is cyclic in a segment, the dynamic behavior constraints are

$$
\begin{aligned}
& i, \hat{i}, j \in I, \quad k \in K, \quad \ell \in M \\
& S_{i}^{\ell}[k]-S_{\hat{i}}^{\ell-1}[k] \geq\left(\delta_{i, j}^{\ell}+\delta_{\hat{i}, j}^{\ell-1}-2\right) \Phi \\
& +T_{i}^{\ell}+L^{\ell} T_{c_{i}}^{\ell}: \quad \ell \in M \backslash\{1\} \\
& S_{i}^{\ell}[k]-S_{i-e}^{\ell}[k]+\beta_{i-e}^{\ell} \Phi \geq T_{i}^{\ell}: i \in I \backslash\{1\}, e=\{1, \ldots, i-1\} \\
& 1-\sum_{j=1}^{n} \delta_{i, j}^{\ell} \varepsilon_{j}^{\ell} \geq \beta_{i}^{\ell} \\
& \alpha_{i}^{\ell} \geq \beta_{i}^{\ell} \\
& S_{1}^{\ell}[k+1]-S_{n}^{\ell}[k] \geq T_{1}^{\ell}: \quad k \in K \backslash\left\{k_{U}^{s}\right\} \\
& S_{1}^{1}[1] \geq T_{1}^{1}
\end{aligned}
$$

CONWIP constraints: Equations (14), (15), and (19) characterize a CONWIP property for the universal model. Providing that the part waiting times in the segment $\ell=1$ are neglected in the stationary state, and the system behaves periodically $\left(C_{g(i, e)}^{m}\left[k+f\left(i, e, N_{i}\right)\right]=C_{g(i, e)}^{m}\right)$, these constraints for part $j$ are rewritten as

$$
\begin{aligned}
& \sum_{e=1}^{N_{i}} C_{g(i, e)}^{m}=\hat{S}_{j}^{m}[k]-\hat{S}_{j}^{1}[k]+\tau_{j}^{1}+T_{r_{j}}^{1} \\
& N_{i}=N+I^{m}(j)-I^{1}(j) \\
& \sum_{i=1}^{n} N_{i}=n N
\end{aligned}
$$

Furthermore, according to the order of releasing actions for $P_{j}$ in the segments $\ell=1$ and $\ell=m\left(I^{1}(j)=\hat{i}\right.$ and $\left.I^{m}(j)=i\right)$, these constraints are modified to

$$
\begin{aligned}
& i, \hat{i}, j \in I, k \in K^{s}, \quad \ell \in M \\
& \left(2-\delta_{i, j}^{m}-\delta_{\hat{i}, j}^{1}\right) \Phi+\sum_{e=1}^{N_{i}} C_{g(i, e)}^{m} \geq S_{i}^{m}[k]-S_{\hat{i}}^{1}[k]+T_{\hat{i}}^{1}+L^{1} T_{c_{\hat{i}}}^{1} \\
& \left(\delta_{i, j}^{m}+\delta_{\hat{i}, j}^{1}-2\right) \Phi+\sum_{e=1}^{N_{i}} C_{g(i, e)}^{m} \leq S_{i}^{m}[k]-S_{\hat{i}}^{1}[k]+T_{\hat{i}}^{1}+L^{1} T_{c_{\hat{i}}}^{1} \\
& \left(2-\delta_{i, j}^{m}-\delta_{\hat{i}, j}^{1}\right) \Phi+N_{i} \geq N+i-\hat{i} \\
& \left(\delta_{i, j}^{m}+\delta_{\hat{i}, j}^{1}-2\right) \Phi+N_{i} \leq N+i-\hat{i} \\
& \sum_{i=1}^{n} N_{i}=n N
\end{aligned}
$$

When $N_{i}(i \in I)$ are known parameters, (34) presents a set of linear constraints that can be devised in linear optimizations models. With $N_{i}$ as variables, these constraints should be linearized as follows (the linearization procedure has been introduced in [17]).

$$
\begin{aligned}
& i, \hat{i}, j \in I, \quad e \in I, \quad k \in K^{s} \\
& C q_{i}+r_{i}+\left(2-\delta_{i, j}^{m}-\delta_{\hat{i}, j}^{1}\right) \Phi \geq S_{i}^{m}[k]-S_{\hat{i}}^{1}[k]+T_{\hat{i}}^{1}+L^{1} T_{\hat{i}_{\hat{i}}}^{1} \\
& C q_{i}+r_{i}+\left(\delta_{i, j}^{m}+\delta_{\hat{i}, j}^{1}-2\right) \Phi \leq S_{i}^{m}[k]-S_{\hat{i}}^{1}[k]+T_{\hat{i}}^{1}+L^{1} T_{c_{\hat{i}}}^{1} \\
& r_{i}=\sum_{e=1}^{n} d_{i, e} \\
& C_{g(i, e)}^{m} \geq d_{i, e} \\
& d_{i, e} \geq C_{g(i, e)}^{m}-\left(1-x_{i, e}\right) C \\
& C x_{i, e} \geq d_{i, e} \\
& x_{i, e} \geq x_{i, e+1}: \quad e \in I \backslash\{n\} \\
& N_{i}=n q_{i}+\sum_{e=1}^{n} x_{i, e} \\
& \left(2-\delta_{i, j}^{m}-\delta_{\hat{i}, j}^{1}\right) \Phi+N_{i} \geq N+i-\hat{i} \\
& \left(\delta_{i, j}^{m}+\delta_{\hat{i}, j}^{1}-2\right) \Phi+N_{i} \leq N+i-\hat{i} \\
& n N=\sum_{i=1}^{n} N_{i}
\end{aligned}
$$

In these constraints $x_{i, e}$ is a binary variable, $q_{i}$ is a positive integer variable, and $\left\{r_{i}, d_{i, e}\right\}$ are positive real variables.

Buffer constraints: Similar to (31) and (33), the constraints $\sum_{e=1}^{N_{i}^{\ell}} C_{g(i, e)}^{\ell}=\hat{S}_{j}^{\ell}[k]-\hat{S}_{j}^{\ell-1}[k]$ for $I^{\ell}(j)=i$ and $n N^{\ell}=$ $\sum_{i=1}^{n} N_{i}^{\ell}$ keep $N^{\ell}$ parts in segment $\ell$. Because $N^{\ell} \leq b^{\ell}$, the relation $\sum_{e=1}^{b^{\ell}} C_{g(i, e)}^{\ell} \geq \hat{S}_{j}^{\ell}[k]-\hat{S}_{j}^{\ell-1}[k]$ forces the segment to always buffer the number of parts lower than the buffer size. 
Considering $I^{\ell-1}(j)=\hat{i}$, this implies

$$
\begin{aligned}
& i, \hat{i}, j \in I, \quad e \in I, \quad \ell \in M \backslash\{1\}, k \in K^{s} \\
& \sum_{e=1}^{b^{\ell}} C_{g(i, e)}^{\ell}+\left(2-\delta_{i, j}^{\ell}-\delta_{\hat{i}, j}^{\ell-1}\right) \Phi \geq S_{i}^{\ell}[k]-S_{\hat{i}}^{\ell-1}[k]
\end{aligned}
$$

Assuming $\sum_{i=1}^{n} C_{i}^{\ell}=C$, the linearized form of (36) for a variable $b^{\ell}$ is developed similar to (35).

$$
\begin{aligned}
& i, \hat{i}, j \in I, \quad e \in I, \quad k \in K^{s} \\
& C q_{i}^{\ell}+r_{i}^{\ell}+\left(2-\delta_{i, j}^{\ell}-\delta_{i, j}^{\ell-1}\right) \Phi \geq S_{i}^{\ell}[k]-S_{\hat{i}}^{\ell-1}[k] \\
& r_{i}^{\ell}=\sum_{e=1}^{n} d_{i, e}^{\ell} \\
& C_{g(i, e)}^{\ell} \geq d_{i, e}^{\ell} \\
& d_{i, e}^{\ell} \geq C_{g(i, e)}^{\ell}-\left(1-x_{i, e}^{\ell}\right) C \\
& C x_{i, e}^{\ell} \geq d_{i, e}^{\ell} \\
& x_{i, e}^{\ell} \geq x_{i, e+1}^{\ell}: \quad e \in I \backslash\{n\} \\
& b^{\ell} \geq n q_{i}^{\ell}+\sum_{e=1}^{n} x_{i, e}^{\ell}
\end{aligned}
$$

Variable constraints: All variables shall be clarified based on their domains such as binary, natural, and real. These variables are defined based on the set of design constraints $D=\{(24),(30),(25),(34),(35),(36),(37)\}$ as follows.

$$
\begin{aligned}
& i \in I, \quad j \in I, \quad e \in I, \quad \ell \in M, k \in K \\
& N, N_{i}, b^{\ell}, q_{i}, q_{i}^{\ell} \in \mathbb{N} \\
& T_{i}^{\ell}, T_{c_{i}}^{\ell}, S_{i}^{\ell}[k], C_{i}^{\ell}, r_{i}, r_{i}^{\ell}, d_{i, e}, d_{i, e}^{\ell} \in \mathbb{R}^{+} \\
& \delta_{i, j}^{\ell}, x_{i, e}, x_{i, e}^{\ell}, \beta_{i}^{\ell} \in\{0,1\}
\end{aligned}
$$

\section{AgILE AND LEAN DESIGN OF AN AMHS}

With the design variables $N, b^{\ell}, T_{c_{i}}^{\ell}, \delta_{i}^{\ell}$, and $\beta_{i}^{\ell}$, the relation (2) can be characterized by the constraints (24), (25), (30), (35), and (37). According to (3) and (11); therefore, the AMHS lean design is formulated by the following model.

$$
\begin{aligned}
& \min \sum_{e=1}^{4} w_{e} \Re_{e} \\
& \text { subject to } \\
& (24),(25),(30),(35),(37),(38) \\
& \alpha_{i}^{\ell} \geq \sum_{j=1}^{n}\left(\delta_{i, j}^{\ell}-\delta_{i, j}^{\ell-1}\right) j: \quad \ell \in M \backslash\{1\} \\
& \alpha_{i}^{\ell} \geq \sum_{j=1}^{n}\left(\delta_{i, j}^{\ell-1}-\delta_{i, j}^{\ell}\right) j: \quad \ell \in M \backslash\{1\} \\
& T_{c}^{U} \geq T_{c_{i}}^{\ell} \geq T_{c}^{L} \\
& T_{c}^{o} \geq T_{c}^{L} \\
& \alpha_{i}^{\ell} \geq 0, T_{c}^{U} \geq 0, T_{c}^{L} \geq 0
\end{aligned}
$$

Here, $T_{c}^{o}$ is a given value (for instance about 10 ) to bound the objective of (39). Solving this optimization problem, the values of the design variables are obtained. These values give important measures to engineers regarding the AMHS, which should satisfy a desired production scenario with the minimum number of resources. Data about part instances (number and types), segments (number and lengths), operation sequences and times, and throughput define a production scenario. These data can be changed due to a customized production manner (agile aspect) or other reasons. Hence, an agile and lean design of an AMHS that can cope with these changes for various production scenarios is highly appreciated.

One design strategy is to anticipate changes over parameters $\left\{n, m, L^{\ell}, \tau_{i}^{\ell}, C\right\}$ and solve (39) for the related production scenarios. For each design variable, this leads to a domain of optimal values satisfying the scenarios. Therefore, the handling resources should be selected so that their utilizations provide a range of flexibilities for part movements, according to the achieved domains.

Hopefully, the number of production scenarios based on variations in $\left\{n, m, L^{\ell}\right\}$ is not so large in practice, because even a highly flexible manufacturing plant has limitations over the range of product types as well as number and position of machines. But the parameters $\left\{\tau_{i}^{\ell}, C\right\}$ are prone to large deviations that introduce a tremendous number of production scenarios. Moreover, these parameters can also be stochastically varied due to some unestimated causes or machine breakdowns. To deal with anticipated and unanticipated variations in $\left\{\tau_{i}^{\ell}, C\right\}$, a worst case analysis can significantly reduce the effort needed to analyze all related production scenarios for these parameters.

The worst case scenario is realized by considering the minimum value of $C$ and maximum potential increases for $\tau_{i}^{\ell}(i \in I)$. For a given production scenario specified by the known data $\left\{n, m, L^{\ell}, \tau_{i}^{\ell}\right\}$, the procedure to obtain an agile and lean design of an AMHS is conducted by the following steps.

1) The minimum value of $C, C^{\infty}$, is achieved, assuming the extreme values $N \rightarrow \infty, v_{i}^{\ell} \rightarrow \infty$, and $b^{\ell} \rightarrow \infty$. These assumptions give

$$
C^{\infty}=\max \left\{\sum_{i=1}^{n} \tau_{i}^{\ell}: \ell \in M\right\}
$$

2) While having the maximum throughput $n / C^{\infty}$, the maximum potential for increasing $\tau_{i}^{\ell}$ is achieved by selecting $C=C^{\infty}$ and solving the optimization model

$$
\begin{aligned}
& \min \sum_{\ell=1}^{4} w_{e} \Re_{e}-w_{5} \sum_{\ell=1}^{m} \sum_{i=1}^{n} T_{i}^{\ell} \\
& \text { subject to } \\
& \text { Design constraints in (39) }
\end{aligned}
$$

In this optimization model, the term $w_{5}$ is a weight coefficient realizing the degree of the agility. For, $w_{5} \geq 1$, the design tendency is to maximize possible increases of operation times, devising the minimum number of handling resources.

\section{CASE STUdy}

In this section, we introduce a case study which includes 4 part instances $(\mathrm{A}, . ., \mathrm{D})$ and 6 machines. The production data 
for this case are illustrated in Table I.

TABLE I

PRODUCTION DATA FOR THE CASE STUDY

\begin{tabular}{rrrrrr}
\hline \hline$M^{\ell}$ & $T_{A}$ & $T_{B}$ & $T_{C}$ & $T_{D}$ & $L^{\ell}$ \\
& {$[\mathrm{s}]$} & {$[\mathrm{s}]$} & {$[\mathrm{s}]$} & {$[\mathrm{s}]$} & {$[\mathrm{m}]$} \\
\hline$M^{1}$ & 5 & 5 & 5 & 5 & 2.6 \\
$M^{2}$ & 0 & 48 & 14 & 42 & 1.6 \\
$M^{3}$ & 17 & 39 & 0 & 35 & 1.1 \\
$M^{4}$ & 36 & 0 & 27 & 37 & 1.1 \\
$M^{5}$ & 9 & 0 & 19 & 48 & 1.6 \\
$M^{6}$ & 5 & 5 & 5 & 5 & 1.1 \\
\hline
\end{tabular}

To conceptually design an AMHS for the plant according to the lean and agile objectives, we employ the model (41). This optimization problem cannot be solved efficiently without using some relaxation methods. We first relax the binary variables for the tunneling actions $\left(\beta_{i}^{\ell} \in[0,1]\right)$ and drop the buffer constraints (37) and related variables from (41). Proceeding in a similar way for (12), we obtain $\Re_{1}^{\text {min }}=$ $6, \Re_{3}^{\text {min }}=-10, \Re_{4}^{\text {min }}=0$, with the assumption that $T_{c}^{L}<=10$ and $C^{\infty}=104$. For $\rho_{e}=1$, this leads to $w_{1}=0.167, w_{3}=0.1$, and $w_{4}=1$. Substituting the weight coefficients in (41) and solving the model with the mentioned relaxations and $w_{5}=1$, the following design parameters are obtained, $N=11, T_{c_{i}}^{\ell}=$ $T_{c}^{L}=T_{c}^{U}=8.68, \beta_{i}^{\ell}=0$, and

$$
\Delta^{1 . .6}=\left(\begin{array}{cccc}
0 & 0 & 1 & 0 \\
0 & 0 & 0 & 1 \\
1 & 0 & 0 & 0 \\
0 & 1 & 0 & 0
\end{array}\right)
$$

Furthermore, using these parameters in

$$
\min b^{h}-w_{5} \sum_{\ell=1}^{m} \sum_{i=1}^{n} T_{i}^{\ell}
$$

subject to

$$
(24),(25),(30),(37),(34),(38)
$$

a lower bound for the buffer size of the specific segment $h$ is obtained, while the constraints on the buffer size of the other segments are not considered in the model. This means that the first line in (37) is modified by including $\ell=h$. Solving (43) gives the buffer capacities $\{3,2,3,3,2\}$ for $b^{2}$ to $b^{6}$, respectively ( $b^{1}$ can be assumed as large as $N$ to buffer all empty carriers).

The design parameters provide important measures for AMHS designers. We can maintain the maximum throughput $\frac{n}{C^{\infty}}=\frac{4}{104}$ and deviations $\sum_{\ell=1}^{m} \sum_{i=1}^{n}\left(T_{i}^{\ell}-\tau_{i}^{\ell}\right)$ (agile aspect), with only 11 carriers, without tunneling actions and changing the scheduling of parts in machines, with the same part velocities $v_{i}^{\ell} \geq 7.5 \frac{\mathrm{m}}{\mathrm{min}}$, and the given buffer sizes. These values are completely within practical ranges for conveyors, pallet systems, and robots. It is possible to use small AGVs and cranes provided that two or three parts are packed as a unit load on these carriers (it is not practical to put $3 \mathrm{AGVs}$ in the buffer with the length $1.1 \mathrm{~m}$ ).

\section{CONCLUSION}

The demands for a minimal number of handling resources (lean design) and their maximal utilization (agile design) emphasize a concurrent structure and control design of an
AMHS in the conceptual phase. To provide this concurrency, a lean and agile design framework has been introduced in this paper. A universal model has been developed to define a set of part movements without concerning AMHS technology. Furthermore, the objective which characterizes optimal part movements according to the lean and agile paradigms has been formulated for the conceptual design phase. Control measures which are obtained according to this objective provide important keys for designers to conceptualize a concurrent design and control of an AMHS.

For big production plants with many part types and machines, the computation time of (41) can dramatically increase due to a large number of binary decision variables. Development of a systematic approach to combine some heuristic and relaxation methods to overcome the model time complexity can be considered as the continuation of this work.

\section{REFERENCES}

[1] M.P. Groover, "Automation, production systems, and computerintegrated manufacturing", Second Edition, Prentice Hall International, 2001.

[2] J.A. Tompkins, J.A. White, Y.A. Bozer, and J.M.A. Tanchoco, "Facilities Planning", Third Edition, John Wiley \& Sons, NJ, 2003.

[3] J.M.A. Tanchoco, "Material flow systems in manufacturing", Chapman and Hall, London, 1994.

[4] H. Tempelmeier, H. Kuhn, "Flexible manufacturing systems: Decision support for design and operation", Wiley Series in Systems Engineering, 1993.

[5] S. Biller, S.P. Marin, S.M. Meerkov, and L. Zhang, "Closed Bernoulli production Lines: Analysis, Continuous Improvement, and Leanness", IEEE Transactions on Automation Science and Engineering, Vol. 6, No. 1, pp. 168-180, 2009.

[6] J. Li and S.M. Meerkov. Production system engineering. Springer, New York, 2009.

[7] B. Pourbabai, "Performance modeling of a closed loop material handling system", European Journal of Operational Research , Vol 32, pp 340-352, 1987.

[8] C.V. Ramamoorthy, G.S. Ho, "Performance evaluation of asynchronous concurrent systems using Petri nets", IEEE Transactions on Software Engineering, Vol.6, pp. 440-449, 1980.

[9] S.P. Sethi, C. Sriskandarajah, S.L. van de Velde, M.Y. Wang, and H. Hoogeveen, "Minimizing makespan in a pallet-constrained flowshop", Journal of Scheduling, Vol. 2, pp. 115-133, 1999.

[10] M.Y. Wang, S.P. Sethi, C. Sriskandarajah, and S.L. van de Velde, "Minimizing makespan in a flowshop with pallet requirements", Inform. Systems Oper. Res. INFOR, No. 35, pp. 277-285, 1997.

[11] F. Chauvet, J.W. Herrmann, and J.M. Proth, "Optimization of cyclic production systems: a heuristic approach", IEEE Transactions on Robotics and Automation, Vol 19, No. 1, pp. 150-154, 2003.

[12] T. Hsu, O. Korbaa b, R. Dupas, and G. Goncalves, "Cyclic scheduling for F.M.S.: Modelling and evolutionary solving approach", European Journal of Operational Research, Vol. 191, pp. 464-484, 2008.

[13] M. Mashaei, B. Lennartson, F. Sannehed, and G. Abbestam, "Optimal design of a decoupled multiple-loop pallet system for flexible cyclic manufacturing plants", CASE, IEEE Conference on Automation Science and Engineering, pp. 433-438, 2010.

[14] P. Solot, "A heuristic method to determine the number of pallets in a flexible manufacturing system with several pallet types", The International Journal of Flexible Manufacturing Systems, Vol.2, pp. 191-216, 1990.

[15] E.H Frazelle, "Material handling: A technology for industrial competitiveness", Material Handling Research Center Technical Report, Georgia Institute of Technology, Atlanta, 1986.

[16] W.J. Hopp, and M.L. Spearman, "Factory physics: Foundations of manufacturing management", 2nd edition, IrwinlMcGraw Hill, NY, 2000.

[17] M. Mashaei and B. Lennartson, "Energy Reduction in a PalletConstrained Flow Shop through On-Off Control of Idle Machines", IEEE Transactions on Automation Science and Engineering, Vol. 10, No. 1, pp. 45-56, 2013. 